\title{
Circadian clock genes, ovarian development and diapause
}

\author{
William E Bradshaw* and Christina M Holzapfel* \\ See research article http://www.biomedcentral.com/1741-7007/8/116
}

\begin{abstract}
Insects, like most organisms, have an internal circadian clock that oscillates with a daily rhythmicity, and a timing mechanism that mediates seasonal events, including diapause. In research published in BMC Biology, lkeno et al. show that downregulation of the circadian clock genes period and cycle affects expression of ovarian diapause in the insect Riptortus pedestris. They interpret these important results as support for Erwin Bünning's (1936) hypothesis that the circadian clock constitutes the basis of photoperiodism. However, their observations could also be the result of pleiotropic effects of the individual clock genes.
\end{abstract}

of the circadian clock in Drosophila and other insects $[3,4]$ but there has been no clear demonstration that the circadian clock, as a functional module, underpins photoperiodism, whose molecular basis remains unknown. There is, however, evidence that the circadian clock gene timeless influences diapause in the dipteran Drosophila [5]. Now, Ikeno et al. [6] report experiments using RNA interference (RNAi) to target two core clock genes in the heteropteran bean bug Riptortus pedestris, showing that inhibition of either cycle or period expression disrupts a circadian rhythm of cuticle deposition, and at the same time affects ovarian diapause. For reasons we shall explain more fully below, they interpret these important results as support for Bünning's hypothesis, while we argue that pleiotropic effects of the cycle gene are likely at play.

\section{Simplified circadian clockworks}

The basic circadian clock of insects functions as a lightsensitive molecular oscillator, incorporating a light-sensitive protein known as CRYPTOCHROME (CRY) and various feedback loops with positively and negatively acting elements. Common to all insect circadian clocks (Figure 1) is the transcription and translation of the genes cycle and clock, whose protein products form a heterodimer (CYC-CLK) that promotes transcription of period (per). In the cytoplasm, the protein encoded by per (PER) interacts with a number of other clock proteins that include TIM (encoded by timeless) and CRY (encoded by cryptochrome). Negative feedback of CYC/CLK activity appears to build through the night but is relieved at dawn when light triggers the degradation of TIM. Due to ancestral gene duplication, there may be two cryptochrome genes in any given insect species - cry 1 and cry 2 (cry2 is called $c r y-m$ in $R$. pedestris). The ancestral circadian clock probably involved CYC as the positiveacting transcriptional activator, CRY1 as the main photoreceptor, and CRY2 as the light-insensitive, negativeacting transcriptional regulator. In Drosophila, PER is the main negative regulator of clock function, but so far, in all insects where cry2 is present and regardless of cryl's 


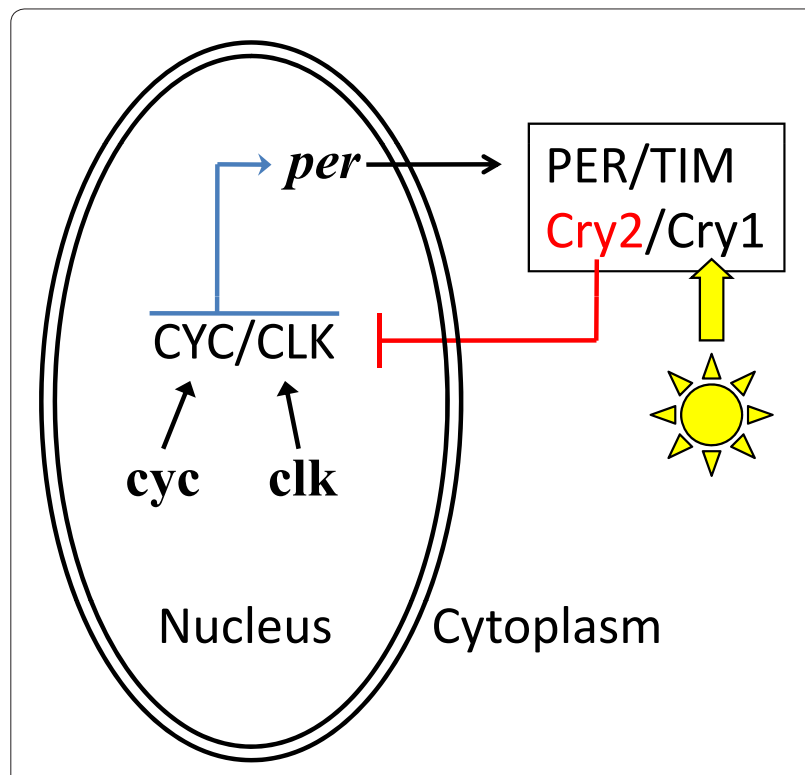

Figure 1. Simplified schematic of circadian clockworks. This schematic is based on the likely state of ancestral insect clocks $[3,4]$ and includes the two genes manipulated by lkeno et al. [6]. The transcription of cycle (cyc) is continuous and that of clock (clk) is rhythmic. The translated proteins (CYC and CLK) form a CYC-CLK heterodimer that promotes the transcription of period (per). per mRNA is transported to the cytoplasm where it is translated into its protein (PER) and forms a complex with the timeless protein (TIM), and two paralogous proteins of cryptochrome (CRY1 and CRY2). CRY1 is the likely photoreceptor that enables the circadian clock to entrain to daily light:dark cycles. CRY2 is a light-insensitive protein that acts as the main transcriptional repressor of the circadian clockworks. In Riptortus pedestris [6], double-stranded RNA directed against cyc (cyc RNAi) effectively reduces cyc and, as expected, per expression; per RNAi effectively reduces per expression and would not be expected to reduce cyc expression. Hence, the principal difference between the effects of cyc and per RNAi at the molecular level would be the level of cyc expression, since per expression is blocked in both cases. Also, the reduced levels of cyc and per that they achieve result in arrhythmicity of daily cuticle deposition, that is, render the circadian clock dysfunctional.

presence, CRY2 plays this role [4]. Thus, in $R$. pedestris, which has cry2 [7], cyc likely acts as the main positive transcriptional activator and $c r y 2$ as the main negative regulator, while per is likely involved in the negatively acting portion of the feedback loop.

\section{The effects of breaking the clock in $R$. pedestris}

Ikeno et al. suppressed the expression of the clock genes cycle and period by injecting bean bugs with doublestranded RNA (RNAi), showing by northerns that this achieved at least partial downregulation of the targeted gene, and also a reduction in per mRNA after $c y c$ RNAi treatment, which would be expected as a secondary consequence of reduced levels of CYC (Figure 1 in [6]). As a read-out for the function of the circadian clock they looked at the layering of the cuticle, which appears as alternating bright and dark layers under polarized light, and is laid down in a rhythm they established as showing the classic features of being regulated by a circadian clock (Figure 1 in [6]). Injection of $c y c$ RNAi resulted in the loss of alternate layering and instead the deposition of a single bright layer. Injection of per RNAi also resulted in the loss of alternate layering, but the outcome was a single dark layer (Figure 3 in [6]). Thus, in both cases there was a loss of circadian rhythm, but with a different phenotypic consequence, and the authors interpret this as an arrest of the clock in two different phases, leading to the activation of distinct downstream cascades.

The remarkable result was that per and $c y c$ RNAi also affected ovarian diapause. Normally $R$. pedestris undergoes an ovarian diapause that is controlled by the length of day (Figure 2). Long days promote ovarian maturation by inhibiting neurohormones from the brain that prevent synthesis or secretion of juvenile hormone from the corpora allata [8]. Short days permit expression of the inhibitory neurohormones and, ultimately, result in nondeveloping ovaries (diapause). When there were significant differences from controls, per RNAi increased the incidence of ovarian development and $c y c$ RNAi decreased the incidence of ovarian development (Figure 4 in [6]). Consistent with these results, and indicating an action on the regulatory cascade leading to diapause at the level of juvenile hormone or upstream, per and cyc RNAi had opposite effects on expression of genes known to be up- or down-regulated by juvenile hormone (Figure 5 in [6]). Furthermore, the application of a juvenile hormone analog (methoprene) restored ovarian development in $c y c$ RNAi bugs (Figure 6 in [6]). Thus, cyc is involved in regulating diapause somewhere between the input of light and the secretion of juvenile hormone in the corpora allata (Figure 2).

\section{Support for Bünning's hypothesis or pleiotropic effects of cyc?}

Ikeno et al. see parallels in their results that lead them to claim support for Bünning's hypothesis. They suggest that having targeted the principal positive regulator of the bean bug's clock $(c y c)$ and a negative regulator (per), they have stopped the circadian clock at different phases of its oscillating cycle. It is unable to oscillate in response to the night-day cycle, but having become stuck in opposing phases, output signals are still delivered to give phenotypes that correspond, in the case of the cuticle, to those normally associated with opposite swings of the night-day pendulum. In the case of diapause, the differences in sensitivity to day length are interpreted as disruption of the photoperiodic timer, with the switch for diapause stuck in either one of two opposing positions. Thus, they see support for Bünning's proposition that the circadian clock mechanism lies at the heart of photoperiodicity. 


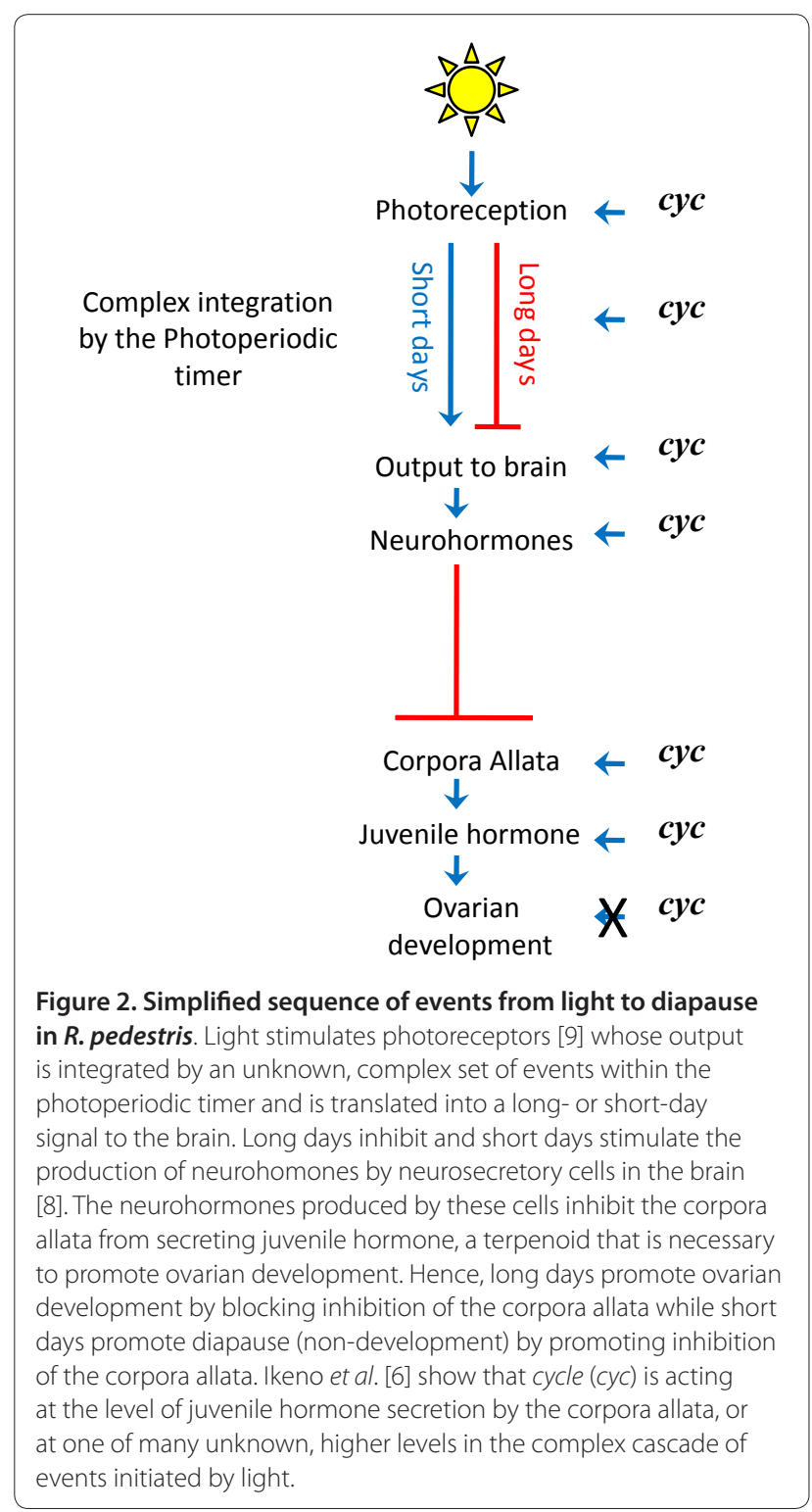

These are, however, speculative suggestions only, venturing beyond the data, and in our view biased by the intrinsic appeal of connecting the circadian clock and photoperiodic time measurement. Based on the data, we would make the following points. First, both RNAi treatments lead to a reduction of per expression, so that any phenotypic differences observed must be attributed largely to the difference in $c y c$ expression. Second, both treatments rendered the circadian clock dysfunctional. Third, all of the phenotypes that varied between the two RNAi treatments did so in the consistent absence of a functional circadian clock. If there is consistently no functional circadian clock in both RNAi treatments, then phenotypic differences between those treatments cannot be ascribed to the circadian clock. We would therefore conclude that the circadian clock as a functional unit (module) does not provide the essential clockworks for photoperiodic time measurement in $R$. pedestris.

Just because the circadian clock is dysfunctional does not mean that individual clock genes have no other pleiotropic effects [9]. The diapause response of bugs exposed to long and short days (Figure 4 in [6]) can be considered in terms of the presence of cyc expression (per RNAi treatment) or absence of $c y c$ expression (cyc RNAi treatment). When $c y c$ is expressed, there is an increase in ovarian development (non-diapause) compared to when cyc is not expressed, regardless of day length. The application of a juvenile hormone analog tells us that $c y c$ is not acting at the level of the ovaries themselves. However, these results do not tell us where $c y c$ is exerting its effect. This could be anywhere in the cascade of events (Figure 2), from the input of light [10] to the secretion of juvenile hormone in the corpora allata. Given that $c y c$ encodes a positive transcriptional regulator, multiple pleiotropic effects are possible, and even likely.

\section{Progress in the understanding of diapause}

In sum, Bünning's hypothesis remains in contention but unproven in insects. Nonetheless, Ikeno et al. [6] have made significant advances in the understanding of the expression of diapause. They have shown in $R$. pedestris that the circadian clock gene $c y c$ plays an important role in ovarian development or diapause, and that it is involved somewhere in the upstream part of the pathway of juvenile hormone and not at the level of the ovaries themselves (Figure 2). Finally, they have added cycle in Heteroptera to timeless in Diptera [5] as core circadian clock genes that also have independent effects - on insect diapause. Their research establishes $c y c$ as an important and interesting focus for future research.

\section{Abbreviations}

cry, cryptochrome (a circadian clock gene); cry1, a Drosophila-like duplicate of cry; cry2, a vertebrate-like duplicate of cry; cyc, cycle (a circadian clock gene); per, period (a circadian clock gene); RNAi, RNA interference; tim, timeless (a circadian clock gene).

\section{Acknowledgments}

We are grateful to the National Science Foundation for their support through grants IOS-0839998 and DEB-0917827 during the preparation of this commentary.

\section{Published: 3 September 2010}

\section{References}

1. Bradshaw WE, Holzapfel CM: What season is it anyway? Circadian tracking vs. photoperiodic anticipation in insects. J Biol Rhythms 2010, 25:155-165.

2. Bünning E: Die endonome Tagesrhythmik als Grundlage der photoperiodischen Reaktion. Ber Dtsch Bot Ges 1936, 54:590-607.

3. Bradley TJ, Briscoe AD, Brady SG, Contreras HL, Danforth BN, Dudley R, Grimaldi, D, Harrison JF, Kaiser JA, Merlin C, Reppert SM, VandenBrooks JM, Yanoviak SP: Episodes in insect evolution. Integr Comp Biol 2009, 49:590-606.

4. Sandrelli F, Costa R, Kyriacou CP, Rosato E: Comparative analysis of circadian clock genes in insects. Insect Mol Bio/ 2008, 17:447-463.

5. Tauber E, Zordan M, Sandrelli F, Pegoraro M, Osterwalder N, Breda C, Daga A, 
Selmin DA, Monger K, Benna C, Rosato E, Kyriacou CP, Costa R: Natural Selection favors a newly derived timeless allele in Drosophila melanogaster. Science 2007, 316:1895-1898.

6. Ikeno T, Tanaka SI, Numata H, Goto SG: Photoperiodic diapause under the control of circadian clock genes in an insect. BMC Bio/ 2010, 8:116

7. Ikeno T, Numata H, Goto SG: Molecular characterization of the circadian clock genes in the bean bug, Riptortus pedestris, and their expression patterns under long- and short-day conditions. Gene 2008, 419:56-61.

8. Simokawa K, Numata H, Shiga S: Neurons important for the photoperiodic control of diapause in the bean bug, Riptortus pedestris. J Comp Physiol A Neuroethol Sens Neural Behav Physiol 2008, 194:751-762.
9. Emerson KJ, Bradshaw WE, Holzapfel CM: Complications of complexity: integrating environmental, genetic and hormonal control of insect diapause. Trends Genet 2009, 25:217-225.

10. Morita A, Numata $\mathrm{H}$ : Distribution of photoperiodic receptors in the compound eyes of the bean bug, Riptortus clavatus. J Comp Physiol A Neuroethol Sens Neural Behav Physiol 1997, 180:181-185.

doi:10.1186/1741-7007-8-115

Cite this article as: Bradshaw WE, Holzapfel CM: Circadian clock genes,

ovarian development and diapause. BMC Biology 2010, 8:115. 